\title{
STANDARD TREATMENT WITH BEVACIZUMAB AS TARGETED THERAPY IN CERVICAL CANCER
}

\author{
Isabel Enríquez-Aceves ${ }^{1}$, Tatiana Galicia-Carmona ${ }^{2,3}$, Jaime A. Coronel-Martínez ${ }^{2,3}$, \\ Raquel Espinosa-Romero ${ }^{3}$, Germán Calderillo-Ruíz ${ }^{3}$; Patricia Cortés-Esteban ${ }^{4}$, \\ AND LUCELY CetinA-PÉreZ ${ }^{2,3 *}$
}

${ }^{1}$ Department of Medical Oncology, Hospital del Instituto de Seguridad y Servicios Sociales de los Trabajadores del Estado (ISSSTE), León, Gto., Mexico; Departments of ${ }^{2}$ Clinical Research and ${ }^{3}$ Medical Oncology, Instituto Nacional de Cancerología, Mexico City; ${ }^{4}$ Department of Medical Oncology, Centro Médico Nacional 20 de Noviembre, Mexico City, Mexico

\begin{abstract}
Metastatic, recurrent, or persistent disease in cervical cancer has a poor prognosis. Historically, this group of patients has had limited treatment options, even with the best cytotoxic treatments (platinum-based chemotherapy [CT] doublets). Therefore, investigating new medications that help improve the patient's quality of life and survival has been essential. Angiogenesis has been shown to play a critical role in tumor cell growth and survival. Bevacizumab is a recombinant humanized monoclonal $\mathrm{G} 1$ immunoglobulin targeted against vascular endothelial growth factor. The combination of CT and bevacizumab is associated with an increase in overall survival as well as in progression-free survival and response rates. (REV INVEST CLIN. 2020;72(4):213-8)
\end{abstract}

Key words: Bevacizumab. Cervical. Cancer. Metastatic antiangiogenic.

\section{INTRODUCTION}

Cervical cancer (CC) remains a health problem in lowand middle-income countries according to the report issued by GLOBOCAN in $2018^{1}$. In Mexico, $80 \%$ of cases are diagnosed at locally advanced stages (IB2 to IVA). The possibility of recurrence is increased according to the initial clinical stage, with recurrence rates in clinical stage IB of $10 \%, 17 \%$ in IIA, $23 \%$ in IIB, $42 \%$ in IIIC, and $72 \%$ in IVA ${ }^{2}$. In addition, between $8 \%$ and $17 \%$ of cases are initially diagnosed in the metastatic stage. Treatment selection in this group of patients will depend on the site of recurrence, whether local, regional, or distant ${ }^{3}$.

In metastatic, recurrent, and persistent stages in patients who are not candidates for surgical management and/or radiotherapy, the choice is systemic treatment ${ }^{3}$ with platinum-based chemotherapy (CT) doublets. The GOG 204 trial assessed the combination of cisplatin with paclitaxel, vinorelbine, gemcitabine, and topotecan and revealed similar efficacy in terms
*Corresponding author:

Lucely Cetina-Pérez

E-mail: lucelycetina.incan@gmail.com
Received for publication: 20-02-2020

Approved for publication: 17-04-2020

DOI: $10.24875 / R I C .20000061$

0034-8376 / (c) 2020 Revista de Investigación Clínica. Published by Permanyer. This is an open access article under the CC BY-NC-ND license (http://creativecommons.org/licenses/by-nc-nd/4.0/). 
of response rates (29\%), progression-free survival (PFS) (5.8 months), and overall survival (OS) (12.9 months), with a tendency for best efficacy in the cisplatin/paclitaxel group ${ }^{4}$.

Five-year survival in the metastatic stage is $16.5 \%$ in comparison with $91.5 \%$ for localized disease ${ }^{5}$. Therefore, despite the benefits of treatment with $\mathrm{CT}$, there was a need for novel treatments to improve survival and quality of life in this group of patients; among the new biological agents, antiangiogenic therapy has shown the greatest benefits. The aim of this article is to analyze the existing publications on the mechanisms behind this therapeutic approach and the benefits and risks of antiangiogenic therapy in advanced CC patients. To this purpose, the NCBI-PubMed and Medline databases were used to search for original articles that reported the results of studies that assessed the effect of anti-vascular endothelial growth factor (anti-VEGF) antibody therapy on response to treatment, toxicity and side effects, tumor recurrence, and OS in CC patients. Other antiangiogenic therapies were also included in the search. Articles were reviewed by authors and evaluated using the GRADE system. Recommendations on the use of antiangiogenic therapy were established according to the quality of the evidence.

\section{ANTIANGIOGENIC THERAPY IN CERVICAL CANCER}

The main cause of CC is human papillomavirus (HPV) latent infection. HPV pathogenic activity hinges on the presence of the $\mathrm{E} 6$ and $\mathrm{E} 7$ proteins: $\mathrm{E} 6$ promotes p53 degradation, while E7 inactivates the retinoblastoma protein. p53 degradation could be responsible for angiogenesis activation through the production of VEGF and downregulation of thrombospondin-1 - a potent angiogenesis inhibitor $-{ }^{6}$. Angiogenesis has a critical function in tumor cell growth and survival, while VEGF plays a highly important role in its control, in tumor growth, and in the development of metastasis ${ }^{7}$.

Some studies have demonstrated the importance of angiogenesis in premalignant lesions of the cervix, with an association between microvessel density and the grade of cervical intraepithelial neoplasia. Cervical samples with high vascularization have been associated with worse survival rates in comparison with poorly vascularized samples (5-year survival of $50 \%$ vs. $65 \%$ ). Furthermore, high levels of VEGF have been associated with CC advanced stages, higher risk of metastasis, and worse PFS and OS data ${ }^{8}$.

\section{BEVACIZUMAB IN CERVICAL CANCER}

Bevacizumab is a recombinant humanized monoclonal G1 immunoglobulin antibody targeted against VEGF (anti-VEGF), clinically developed for various oncological entities ${ }^{9}$. A retrospective study of six heavily pretreated CC patients assessed the use of bevacizumab in combination with 5-fluorouracil or capecitabine. Clinical benefit was observed in $67 \%$ of patients: one complete response (CR), 17\%, one partial response (PR), $17 \%$, and two patients with stable disease (SD), $33 \%$, with a mean time to progression of 4.3 months. In addition, the combination was well tolerated, with anemia being the most common toxic effect. Grade 3 anemia occurred in $17 \%$ of patients, Grade 2 in $33 \%$, and Grade 1 in $50 \%{ }^{10}$.

Bevacizumab has been assessed in Phase II studies. One of them analyzed its efficacy and tolerability in 46 patients with recurrent $\mathrm{CC}^{11}$. PR was achieved in $10.9 \%$ of patients and PFS in $23.9 \%$, for a minimum of 6 months. Mean response duration was 6.21 months, PFS was 3.4 months ( $95 \% \mathrm{Cl}: 2.53-4.53$ ), and OS 7.29 months (95\% Cl: 6.11-10.41 months). Among the main Grade 3 or 4 , adverse events (AEs) caused by bevacizumab, hypertension, pain, and thromboembolism, and other events of hematological, gastrointestinal, genitourinary, and renal origin were reported.

Another Phase II trial evaluated the combination of $\mathrm{CT}$ with topotecan and cisplatin plus bevacizumab in the treatment of recurrent or persistent carcinoma of the cervix ${ }^{12}$. Twenty-six patients were evaluated, one of whom achieved CR and 8 PR, with a mean response duration of 4.4 months, and 10 patients had SD with a duration of 2.2 months. The probability of PFS at 6 months was $59 \%$ ( $80 \% \mathrm{Cl}: 46-70 \%)$, and for the set of patients, mean PFS was 7.1 months (80\% Cl: 4.7-10.1 months) and mean OS was 13.2 months ( $80 \%$ IC: $8.0-15.4$ months). Treatment delay of at least one cycle due to toxicity was recorded in $59 \%$ of patients, while $78 \%$ had to be 
hospitalized to manage toxicity. Reported hematologic AEs (Grades 3-4) included thrombocytopenia ( $82 \%)$, leukopenia (74\%), anemia (63\%), and neutropenia ( $56 \%$ ). Red blood cell transfusion was required by $78 \%$ of patients and platelet transfusion by $30 \%$. Other non-hematologic Grades 3-4 toxicities were metabolic abnormalities (44\%), pain (33\%), and genitourinary or renal complications (30\%). This study showed that the combination of topotecan plus cisplatin with bevacizumab is active but highly toxic.

These studies set the standard for conducting the GOG 240 Phase III trial that, in 2014, led to the approval of bevacizumab by the U.S. Food and Drug Administration for the treatment of advanced CC. The study assessed bevacizumab effectiveness with a combination of $\mathrm{CT}$ in patients with recurrent, persistent, or metastatic CC. Four-hundred and fifty-two patients were randomized to receive CT with or without bevacizumab ( $15 \mathrm{mg} / \mathrm{kg}$ day 1 ). CT consisted of 21-day cycles with cisplatin ( $50 \mathrm{mg} / \mathrm{m}^{2}$ day 1 ) plus paclitaxel (135 or $175 \mathrm{mg} / \mathrm{m}^{2}$ day 1 ) or topotecan $\left(0.75 \mathrm{mg} / \mathrm{m}^{2}\right.$ days $\left.1-3\right)$ plus paclitaxel $\left(175 \mathrm{mg} / \mathrm{m}^{2}\right.$ day 1 ). The study data showed that the addition of bevacizumab to $\mathrm{CT}$ was associated with an increase in OS (17.0 vs. 13.3 months; HR: 0.71 ; $95 \%$ Cl: $0.54-$ $0.95 ; \mathrm{p}=0.004)$ and in response rates ( $48 \%$ vs. $36 \%$; $\mathrm{p}=0.008)$ in comparison with CT alone. Bevacizumab was also associated with an increased incidence of AEs, which included Grade $\geq 2$ hypertension ( $25 \%$ vs. $2 \%$ ), Grade $\geq 3$ thromboembolic events ( $8 \%$ vs. $1 \%$ ), and Grade $\geq 3$ gastrointestinal fistulae ( $3 \%$ vs. $0 \%$ ). There was no quality of life deterioration according to the Functional Assessment of Cancer Therapy-Trial Outcome Index questionnaire for CC, which was applied to patients from cycle 1 through cycle 9 ( $98.75 \%$ $\mathrm{Cl}:-4.1-1.7 ; \mathrm{p}=0.30)^{13}$.

In the GOG 240 final analysis, the benefit was still a greater OS in patients treated with CT plus bevacizumab in comparison with CT alone (16.8 vs. 13.3 months; HR: $0.77 ; 95 \% \mathrm{Cl}: 0.62-0.95 ; \mathrm{p}=0.0068$ ). When analyzing by $\mathrm{CT}$, bevacizumab was observed to be able to increase OS only when added to cisplatin plus paclitaxel (17.5 vs. 15.0 months), while there was no difference when added to topotecan plus paclitaxel (16.2 vs. 12.0 months). OS in patients who had not previously received pelvic radiotherapy was higher in the group treated with bevacizumab in comparison with the group treated with CT alone (24.5 vs. 16.8 months), although the differences were not significant (HR: 0.64; $95 \% \mathrm{Cl}: 0.37-1.10 ; p=0.11$ ). Post-progression OS also did not show significant differences between treatments (8.4 vs. 7.1 months; HR: 0.83 ; $95 \% \mathrm{Cl}$ : $0.66-$ $1.05 ; \mathrm{p}=0.06$ ). PFS final data also revealed that adding bevacizumab to $\mathrm{CT}$ decreased the risk of progression by approximately $32 \%$ in comparison with CT alone (8.2 vs. 6.0 months; HR: 0.68; $95 \% \mathrm{Cl}$ : 0.56-0.84; $\mathrm{p}=0.0002$ ). The overall response rate (ORR) was $49 \%$ in patients treated with CT plus bevacizumab and $36 \%$ for CT alone ( $p=0.003), 50 \%$ and $46 \%$ in patients treated with cisplatin plus paclitaxel with and without bevacizumab, respectively, and $48 \%$ and $25 \%$ for those who received topotecan plus paclitaxel with and without bevacizumab $(p=0.0004)$. The most commonly reported AEs were the development of fistulas. The incidence of fistulas of any grade was 15\% in patients treated with CT plus bevacizumab versus $1 \%$ in those treated with $\mathrm{CT}$ alone and Grade 3 fistulas, $6 \%$ and $<1 \%$, respectively ${ }^{14}$.

The incorporation of bevacizumab to the treatment of recurrent, persistent, or metastatic CC represents a huge advance since it shows a survival benefit; however, we lack a biomarker to define which group of patients are candidates for treatment with bevacizumab.

The original impetus to study poor prognosis markers in advanced CC was to identify a priori patients who were unlikely to respond to conventional cytotoxic therapy in an effort to avoid the administration of futile treatment ${ }^{15}$. The Moore criteria were identified in the platinum or cytotoxic era when antiangiogenic agents were not yet used in randomized clinical trials in patients with $\mathrm{CC}^{16-18}$.

The application of the Moore criteria to the entire GOG-240 study population had the purpose of prospectively analyze previously identified clinical prognostic factors, to validate a score using said criteria: performance status, pelvic disease, ethnicity, disease-free interval $<1$ year, and previous exposure to cisplatin. Risk categories included low risk (0-1 factor), intermediate risk (2-3 factors), and high risk (4-5 factors). The benefit of receiving bevacizumab was observed to be superior in moderateand high-risk patients, with an increase in OS of up to 5.8 months $^{19,20}$. A retrospective study assessed the eligibility of patients with recurrent and 
metastatic CC with a follow-up of more than 10 years and found that the main reasons for exclusion from treatment were transvaginal active bleeding and poor renal function ${ }^{21}$.

The combination of carboplatin and paclitaxel plus bevacizumab in metastatic disease can be considered an alternative treatment option in patients who are not candidates for cisplatin, which is evidence provided by the Japanese study that assessed carboplatin non-inferiority versus cisplatin 22 .

The preliminary report of the CECILIA open-label Phase II study, which is assessing the efficacy and safety of the combination with carboplatin area under the curve 5 every 3 weeks and paclitaxel at $175 \mathrm{mg} / \mathrm{m}^{2}$ plus bevacizumab $15 \mathrm{mg} / \mathrm{kg}$ every 3 weeks in 150 patients with metastatic, recurrent, or persistent CC, suggests that the combination is safe, with a similar risk of fistulae to that in the GOG 240 trial $^{23}$.

With these data, the National Comprehensive Cancer Network, the European Society for Medical Oncology (ESMO) and the American Society of Clinical Oncology (ASCO) guidelines recommend the use of bevacizumab combined with $\mathrm{CT}$ in patients with $\mathrm{CC}$, in metastatic or recurrent disease with a high degree of recommendation and high quality of evidence ${ }^{24-26}$.

\section{OTHER ANTIANGIOGENIC AGENTS AND TARGETED THERAPIES IN THE TREATMENT OF CERVICAL CANCER}

Additional targeted strategies include other antiangiogenic agents (pazopanib, lapatinib, sunitinib, or cediranib), which have not been approved for standard management.

Pazopanib is a tyrosine kinase inhibitor (TKI) that inhibits the signal transduction of multiple VEGF receptors, platelet-derived growth factor receptors (PDGFR), and stem cell receptor (c-KIT). Lapatinib is a TKI that inhibits epidermal growth factor receptor (EGFR) and HER2/neu. One study assessed 230 patients with advanced CC, who were randomized to receive pazopanib or lapatinib monotherapy. Pazopanib showed better PFS (18.1 vs. 17.1 weeks; HR: $0.66 ; 90 \% \mathrm{Cl}: 0.45-0.91 ; \mathrm{p}<0.013$ ) and OS (50.7 vs.
39.1 weeks; HR: $0.67 ; 90 \% \mathrm{Cl}: 0.46-0.99 ; \mathrm{p}=0.045$ ) than lapatinib 27.

Sunitinib is a TKI that inhibits several VEGF receptors, the PDGFR receptor, and c-kit. A Phase II trial with 19 patients showed a 3.5-month PFS $(95 \% \mathrm{Cl}$ : 2.6-7 months) with no documented treatment response and high morbidity with $26.3 \%$ of cases developing fistulae, which led to rule out sunitinib monotherapy in CC (19). Cediranib is a TKI that inhibits several VEGF receptors. A Phase II study compared cediranib treatment versus placebo in 69 patients with recurrent or metastatic CC. PFS was 35 weeks for cediranib and 30 weeks for placebo (HR: $0.61 ; 80 \% \mathrm{Cl}$ : $0.41-0.89 ; p=0.046$ ), and ORR was $66 \%$ and $42 \%$. Cediranib was associated with a higher incidence of Grade 3 or 4 neutropenia than placebo (31\% vs. $9 \%$, $\mathrm{p}=0.019$ ); in addition, $50 \%$ and $34 \%$ of patients experienced Grades 2-4 diarrhea and hypertension, respectively, with no impact on OS ${ }^{28}$.

Another pathway implicated in CC is that of EGFR which, like angiogenesis, plays an important role in CC pathogenesis. EGFR overexpression in CC is considered a factor of poor prognosis in terms of survival and response to $\mathrm{CT}^{29}$. With the above evidence, EGFR blockade was considered to be a potential approach that could be used in the treatment of CC. Pilot and Phase II trials were conducted with EGF pathway TKI such as erlotinib and gefitinib. As a single agent, erlotinib was shown to be inefficient, while gefitinib, as second- or third-line treatment, showed no objective response in patients with metastatic disease ${ }^{30,31}$.

Cetuximab and nimotuzumab, which are anti-EGFR antibodies, either alone or in combination with $\mathrm{CT}$, showed discrete activity in small pilot studies or Phase II clinical trials and although these agents were well tolerated, there was no clinical response ${ }^{32,33}$.

\section{CONCLUSIONS}

Angiogenesis is a process proven to be determinant for tumor growth and invasion. Therefore, antiangiogenic therapy with bevacizumab, targeted against VEGF, shows promise to prevent tumor recurrence and the development of advanced disease. The 
combination of CT and bevacizumab is the best approach in advanced CC patients, with a demonstrated increase in OS as well as in PFS and response rates.

\section{RECOMMENDATIONS}

1. In patients with recurrent, persistent, or metastatic CC, the use of bevacizumab in combination with platinum-based CT is indicated. Quality of evidence: (GRADE) high. Strength of recommendation: strong in favor of its use.

2. In patients with transvaginal active bleeding, poor renal function, and/or poor performance status, the use of bevacizumab may be considered. Quality of evidence: (GRADE) moderate. Strength of recommendation: weak in favor of its use.

3. In patients with recurrent, persistent, or metastatic CC, the use of carboplatin is non-inferior versus cisplatin; therefore, both are options as first line of treatment. Quality of evidence: (GRADE) high. Strength of recommendation: strong in favor of its use.

4. In patients who have previously received cisplatin concomitant with RT, it is preferred to use carboplatin in metastatic disease. Quality of evidence: (GRADE) high. Strength of recommendation: strong in favor of its use.

5. The use of bevacizumab combined with CT in patients with CC, in metastatic or recurrent disease, is recommended. Quality of evidence: (GRADE) high. Strength of recommendation: strong in favor of its use.

\section{REFERENCES}

1. Bray F, Ferlay J, Soerjomataram I, Siegel RL, Torre LA, Jemal A. Global cancer statistics 2018: GLOBOCAN estimates of incidence and mortality worldwide for 36 cancers in 185 countries. CA Cancer J Clin. 2018;68:394-424.

2. Peiritti M, Zapardiel I, Zanagnolo V, Landoni F, Morrow CP, Maggioni A. Management of recurrent CC: a review of the literature. Surg Oncol. 2012;21:e59-66.

3. Sociedad Mexicana de Oncología. Primer consenso nacional de prevención, diagnóstico y tratamiento del cáncer cervicouterino. GAMO. 2014;13 Suppl 4:1-106.
4. Monk BJ, Sill MW, McMeekin DS, Cohn DE, Ramondetta LM, Boardman $\mathrm{CH}$, et.al. Phase III trial of four cisplatin-containing doublet combinations in stage IVB, recurrent, or persistent cervical carcinoma: a gynecologic oncology group study. J Clin Oncol. 2009;27:4649-55

5. Ferlay J, Steliarova-Foucher E, Lortet-Tieulent J, Rosso S, Coebergh JW, Comber H, et al. Cancer incidence and mortality patterns in Europe: estimates for 40 countries in 2012. Eur J Cancer. 2013;49:1374-403.

6. Tomao F, Papa A, Rossi L, Zaccarelli E, Caruso D, Zoratto F, et al. Angiogenesis and antiangiogenic agents in cervical cancer. Onco Targets Ther. 2014;7:2237-48

7. Kerbel RS. Tumor angiogenesis. N England J Med. 2008; 358:2039-49.

8. Eskander RN, Tewari KS. Development of bevacizumab in advanced cervical cancer: pharmacodynamic modelling, survival impact and toxicology. Future Oncol. 2015;11:909-22.

9. Fisher C, Schefter T. Profile of bevacizumab and its potencial in the treatment of cervical cancer. Onco Targets Ther. 2015; 8:3425-31.

10. Wright JD, Viviano D, Powell MA, Gibb RK, Mutch DG, Grigsby PW, et al. Bevacizumab combination therapy in heavily pretreated, recurrent cervical cancer. Gynecol Oncol. 2006; 103:489-93.

11. Monk BJ, Sill MW, Burger RA, Gray HJ, Buekers TE, Roman LD. Phase II trial of bevacizumab in the treatment of persistent or recurrent squamous cell carcinoma of the cervix: a gynecologic oncology group study. J Clin Oncol. 2009;27:1069-74.

12. Zighelboim I, Wright JD, Gao F, Case AS, Massad LS, Mutch DG, et al. Multicenter phase II trial of topotecan, cisplatin and bevacizumab for recurrent or persistent cervical cancer. Gynecol Oncol. 2013;130:64-8.

13. Tewari KS, Sill MW, Long HJ 3rd, Penson RT, Huang H, Ramondetta LM, et al. Improved survival with bevacizumab in advanced cervical cancer. N Engl J Med. 2014;370:734-43.

14. Tewari KS, Sill MW, Penson RT, Huang H, Ramondetta LM, Landrum LM, et al. Bevacizumab for advanced cervical cancer: final overall survival and adverse event analysis of a randomised, controlled, open-label, phase 3 trial (gynecologic oncology group 240). Lancet. 2017;390:1654-63.

15. Tewari KS, Monk BJ. Recent achievements and future developments in advanced and recurrent cervical cancer: trials of the gynecologic oncology group. Semin Oncol. 2009;36:170-80.

16. Tewari KS, Monk BJ. The rationale for the use of non-platinum chemotherapy doublets for metastatic and recurrent cervical carcinoma. Clin Adv Hematol Oncol. 2010;8:108-15

17. Tewari KS. Patients with metastatic/recurrent cervical cancer should be treated with cisplatin plus paclitaxel. Expert panel. Clin Ovarian Cancer. 2011;4:90-3.

18. Moore DH. Chemotherapy for recurrent cervical carcinoma. Curr Opin Oncol. 2006;18:516-59.

19. Moore DH, Tian C, Monk BJ, Long HJ, Omura GA, Bloss JD. Prognostic factors for response to cisplatin-based chemotherapy in advanced cervical carcinoma: a gynecologic oncology group study. Gynecol Oncol. 2010;116:44-9.

20. Tewari KS, Sill MW, Monk BJ. Prospective validation of pooled prognostic factors in women with advanced cervical cancer treated with chemotherapy with/without bevacizumab: NRG oncology/GOG study. Clin Cancer Res. 2015;21:5480-7.

21. Skelton W, Castagno J, Cardenas-Goicoechea J, Daily K, Yeung A, Markham MJ. Bevacizumab eligibility in patients with metastatic and recurrent cervical cancer: a retrospective review. Clin Med Insights Oncol. 2018;12:1-5.

22. Kitagawa R, Katsumata N, Shibata T, Kamura T, Kasamatsu T, Nakanishi T, et al. Paclitaxel plus carboplatin versus paclitaxel plus cisplatin in metastatic or recurrent cervical cancer: the open-label randomized phase III trial JCOG0505. J Clin Oncol. 2015;33:2129-35.

23. Arredondo A, Colombo N, Deostri LM, McCormack M, Rodrigues AN, Donica $M$, et al. Preliminary results from CECILIA an open-label global safety study of bevacizumab (BEV), carboplatin $(C)$ and paclitaxel $(P)$ therapy for metastatic, recurrent, or persistent cervical cancer (CC). J Clin Oncol. 2018;36 Suppl 15:5528

24. NCCN Clinical Practice Guidelines in Oncology. NCCN Guidelines $^{\oplus}$. Cervical Cancer. Version 4. United States: NCCN Clinical Practice Guidelines in Oncology; 2019.

25. Marth C, Landoni F, Mahner S, McCormack M, Gonzalez-Martin A, Colombo N, et al. Cervical cancer: ESMO clinical practice guidelines for diagnosis, treatment and follow-up. Ann Oncol. 2017;28 Suppl 4:72-83. 
26. Chuang LT, Temin S, Camacho R, Dueñas-Gonzalez A, Feldman $S$, Gultekin $M$, et al. Management and care of women with invasive cervical cancer: American society of clinical oncology resource-stratified clinical practice guideline. J Glob Oncol. 2016:2:311-40.

27. Monk BJ, Mas Lopez L, Zarba JJ, Oaknin A, Tarpin C, Termrungruanglert W, et al. Phase II, open-label study of pazopanib or lapatinib monotherapy compared with pazopanib plus lapatinib combination therapy in patients with advanced and recurrent cervical cancer. J Clin Oncol. 2010;28:3562-9.

28. Mackay HJ, Tinker A, Winquist E, Thomas G, Swenerton K, Oza A, et al. A phase II study of sunitinib in patients with locally advanced or metastatic cervical carcinoma: NCIC CTG Trial IND.184. Gynecol Oncol. 2010;116:163-7.

29. Symonds RP, Gourley C, Davidson S, Carty K, McCartney E, Rai $D$, et al. Cediranib combined with carboplatin and paclitaxel in patients with metastatic or recurrent cervical cancer (CIRCCa): a randomised, double-blind, placebo-controlled phase 2 trial. Lancet Oncol. 2015;16:1515-24.
30. Schilder RJ, Sill MW, Lee YC, Mannel R. A phase II trial of erlotinib in recurrent squamous cell carcinoma of the cervix: a gynecologic oncology group study. Int J Gynecol Cancer. 2009;19:929-33.

31. Goncalves A, Fabbro M, Lhommé C, Gladieff L, Extra JM, Floquet $A$, et al. A phase II trial to evaluate gefitinib as second-or third-line treatment in patients with recurring locoregionally advanced or metastatic cervical cancer. Gynecol Oncol. 2008; 108:42-6.

32. Santin AD, Sill MW, McMeekin DS, Leitao MM Jr., Brown J, Sutton GP, et al. Phase II trial of cetuximab in the treatment of persistent or recurrent squamous or non-squamous cell carcinoma of the cervix: a gynecologic oncology group study. Gynecol Oncol. 2011;122:495-500

33. Cetina L, Crombet T, Jiménez-Lima R, Zapata $S$, Ramos M, Avila $S$, et al. A pilot study of nimotuzumab plus single agent chemotherapy as second-or third-line treatment or more in patients with recurrent, persistent or metastatic cervical cancer. Cancer Biol Ther. 2015;16:684-9. 